\title{
Cranial irradiation inhibits hippocampal neurogenesis via DNMT1 and DNMT3A
}

\author{
SHENGJUN JI ${ }^{1}$, XIN DING $^{2}$, JIANG JI ${ }^{2}$, HAOHAO WU ${ }^{2}$, RUI SUN ${ }^{2}$, \\ XIAOYANG $\mathrm{LI}^{2}$, LIYUAN ZHANG ${ }^{2}$ and YE TIAN ${ }^{2}$ \\ ${ }^{1}$ Cancer Center, Nanjing Medical University Affiliated Suzhou Hospital, Suzhou, Jiangsu 215001; \\ ${ }^{2}$ Department of Radiotherapy and Oncology, The Second Affiliated Hospital of \\ Soochow University, Suzhou, Jiangsu 215004, P.R. China
}

Received April 4, 2016; Accepted November 16, 2017

DOI: $10.3892 / \mathrm{ol} .2017 .7643$

\begin{abstract}
Impairment of neurogenesis in the hippocampus following whole-brain irradiation is the most important mechanism of radiation-induced cognitive dysfunction. However, the underlying mechanism remains obscure, meaning an ideal therapeutic target has not been identified. Evidence indicates that DNA methylation in neurons regulates synaptic plasticity and neuronal network activity. In the present study, the expression of DNA methyltransferases (DNMTs) in the hippocampus was analyzed to investigate their potential function in radiation-induced neurogenesis impairment. Sprague-Dawley rats were used throughout the present study, apportioned to the following groups: Control, radiation only, zebularine (a DNMT inhibitor) only, and radiation and zebularine together. Immunofluorescence staining revealed that radiation inhibited cellular proliferation and dendritic growth within new neurons of the hippocampus. In addition, western blot analysis demonstrated lower expression levels of DNMT1 and DNMT3A protein following radiation treatment compared with that in the non-irradiated control. Furthermore, compared with the radiation-only group, the radiation and zebularine group had significantly lower cell proliferative abilities, dendritic growth, and DNMT1 and DNMT3A protein levels. The results of the present study indicated that DNMT1 and DNMT3A may be involved in the pathogenesis of whole-brain radiation-induced neurogenesis impairment.
\end{abstract}

\section{Introduction}

Radiation therapy has long been an indispensable treatment for head and neck cancer, and primary and secondary brain

Correspondence to: Dr Ye Tian, Department of Radiotherapy and Oncology, The Second Affiliated Hospital of Soochow University, 1055 San Xiang Road, Suzhou, Jiangsu 215004, P.R. China

E-mail:dryetian@126.com

Key words: radiation, hippocampus, DNA methyltransferase, neurogenesis tumors, which may provide long-term survival benefits for patients (1-3). However, acute and chronic radiation-induced cognitive impairment is a major reason for limiting radiotherapeutic dosage $(2,4)$. This cognitive impairment has a diverse character, but is typically comprised of deficits in hippocampal-dependent functions, such as learning, memory and spatial information processing (5). Numerous studies have demonstrated that irradiation-induced cognitive impairments are associated with decreases in neurogenesis within the hippocampus (5-7).

Granule neurons in the dentate gyrus, which are generated throughout life, subsequently become functionally integrated into the hippocampal circuitry (8). Axons and dendrites are the anatomical bases of synaptic contact $(9,10)$. In humans, progenitor cells in the dentate gyrus are particularly vulnerable to ionizing radiation, even at low doses (11). Irradiation has been demonstrated to reduce the number of proliferating cells in the dentate gyrus of rodents (12); however, the mechanism underlying the neurotoxic effects of radiation has not been definitively identified.

Epigenetic mechanisms, including histone modifications and DNA methylation, appear to contribute to the expression of neuronal genes involved in learning and memory within mouse models; however, there is evidence that histone deacetylase inhibitors promote the recovery of learning and memory (13). A previous study determined that whole-brain irradiation (WBI) was associated with cognitive deficit in Sprague Dawley rats, a reduction in histone $\mathrm{H} 3$ acetylation in the hippocampus and the long-term impairment of neurogenesis in the dentate gyrus (7). However, the function of DNA methylation in the adult nervous system remains unclear.

Epigenetic alterations via DNA methylation are associated with synaptic plasticity, learning and memory (14). DNA methylation, which is catalyzed by DNA methyltransferase 1 (DNMT1), DNMT3A and DNMT3B, prevents the binding of transcription factors to promoter sequences (15). DNA methylation in adult neurons may be crucial for the transcriptional regulation of genes involved in memory formation (16). Evidence indicates that DNA methylation in neurons regulates synaptic plasticity, as well as neuronal network activity. A prior study demonstrated that DNMT1 and DNMT3A double-knockout mice revealed abnormal long-term synaptic 
plasticity in the hippocampal CA1 region, leading researchers to conclude that these DNA methyltransferases are required for synaptic plasticity, learning and memory (17). In addition, DNMT3A overexpression increased the dendritic spine density of nucleus accumbens neurons in mice (18). These findings indicated that DNMTs may be crucial to hippocampal-dependent memory consolidation and neurogenesis in the dentate gyrus. However, alterations in DNMT1 and DNMT3A that may occur following radiation require further investigation.

The present study evaluated the effects of WBI on the protein expression levels of DNMT1, DNMT3A and DNMT3B in the hippocampus, and investigated whether these effects were associated with the radiation-induced impairment of neurogenesis.

\section{Materials and methods}

Study design. Sprague Dawley rats were randomly apportioned to the following treatment groups ( $\mathrm{n}=10 / \mathrm{group}$ ): Control, radiation only, zebularine only, or radiation combined with zebularine (radiation + zebularine). A total of 5 rats were used for immunofluorescence staining and 5 rats were used for western blot.

Each rat received a twice-daily intraperitoneal injection of bromo-deoxyuridine (BrdU; $50 \mathrm{mg} / \mathrm{kg}$ body weight; Sigma-Aldrich; Merck KGaA, Darmstadt, Germany) for 4 days prior to radiation exposure. Then, rats in the radiation only group and the radiation + zebularine group received whole brain irradiation. Rats of the zebularine-only and radiation with zebularine groups received zebularine $(20 \mathrm{mg} / \mathrm{kg}$, Sigma-Aldrich; Merck KGaA) intracoelomic injection with a fine needle via the skin following irradiation. All rats were sacrificed on day 7 following radiation exposure. Immunofluorescence staining and western blotting were used to investigate the effects of WBI on cell proliferation, dendritic growth, and DNMT1 and DNMT3A protein levels.

Animals. A total of 40 healthy male Sprague-Dawley rats (150-200 g) were obtained from the Medical Experimental Animal Center of Soochow University (Suzhou, China). All the rats were housed together, 3-4 animals per cage, at $\sim 24^{\circ} \mathrm{C}$, with ad libitum access to tap water and food. They were kept under natural light in $12 \mathrm{~h}$ cycles. The procedures involving animals and their care were conducted in accordance with the Soochow University Medical Experimental Animal Care Guidelines, which comply with national policies for the ethical use of animals (Regulation of the Administration of Laboratory Animals, order no. 638 of the State Council). The present study was approved by the Ethics Committee of the National Drug Clinical Trial Institution of The Second Affiliated Hospital of Soochow University (Suzhou, China).

Radiation procedure. For radiation treatment, rats were anesthetized with an intraperitoneal injection of $1 \mathrm{ml} / 100 \mathrm{~g}$ (360 mg/kg body weight) chloral hydrate, and placed in a prone position in a linear accelerator (SL 18, Philips UK Ltd., Guildford, UK). Each rat in the radiation-only group and the radiation + zebularine group received a single dose of $10 \mathrm{~Gy}$ from a 4-MeV electron beam, as described previously $(7,19)$.
Drug treatments. Prior to radiation treatments, each rat received an intraperitoneal injection of BrdU; $50 \mathrm{mg} / \mathrm{kg}$ body weight; Sigma-Aldrich; Merck KGaA), twice daily at $8 \mathrm{~h}$ intervals, for 4 days. The DNA methylation inhibitor zebularine was first dissolved in dimethyl sulfoxide, and then diluted in saline. A dose of $20 \mathrm{mg} / \mathrm{kg}$ was administered by intracoelomic injection with a fine needle via the skin; this dose was non-toxic and did not affect the general condition of the rats in the present study. All the animals were sacrificed 7 days after WBI.

Immunohistochemistry. Rats were deeply anaesthetized with an intraperitoneal injection of $1 \mathrm{ml} / 100 \mathrm{~g}(360 \mathrm{mg} / \mathrm{kg}$ body weight) chloral hydrate 7 days after WBI. Then, they were treated with $100 \mathrm{ml} 0.9 \% \mathrm{NaCl}$ via transcardial reperfusion at $4^{\circ} \mathrm{C}$ to flush out the blood, immediately followed by $500 \mathrm{ml} 4 \%$ paraformaldehyde (PFA) in PBS at $4^{\circ} \mathrm{C}$ for $30 \mathrm{~min}$ (pH 7.4). Brains were then carefully extracted, placed in $4 \%$ PFA for overnight fixation at $4^{\circ} \mathrm{C}$ and then transferred to $30 \%$ sucrose at $4^{\circ} \mathrm{C}$ until embedding. Subsequently, brain tissues (30-mm-thick frozen sections) were cut using a cryostat. Immunofluorescence staining was conducted in accordance with previously described procedures (7). Briefly, the sections were treated with $2 \mathrm{M} \mathrm{HCl}$ at $37^{\circ} \mathrm{C}$ to denature DNA and then washed in $1 \mathrm{X}$ Tris-buffered saline $\mathrm{pH} 8.5$ to neutralize the acid. The sections were then incubated in $5 \%$ bovine serum albumin (Beyotime Institute of Biotechnology, Haimen, China) and 5\% Triton X-100 in PBS for $1 \mathrm{~h}$ at room temperature.

Tissue sections were incubated with primary antibodies at $4^{\circ} \mathrm{C}$ for $24 \mathrm{~h}$, followed by a fluorescent secondary antibody for $2 \mathrm{~h}$; the tissues were then washed in PBS 3 times. Cell nuclei were stained with DAPI (Beyotime Institute of Biotechnology) at room temperature for $5 \mathrm{~min}$ prior to mounting on glycerol-treated slides. Analysis of tissue staining was performed using a confocal laser-scanning microscope (Olympus Corporation, Tokyo, Japan) with x10 objective. The excitation wavelength was as follows: Green, $495 \mathrm{~nm}$; red, $590 \mathrm{~nm}$ and blue, $358 \mathrm{~nm}$.

The antibodies and working concentrations of primary antibodies were: Rat anti-doublecortin (DCX; 1:100; cat no. D9818; Sigma-Aldrich; Merck KGaA); mouse anti-neuronal nuclear antigen (NeuN; 1:50; cat no. mab377; Chemicon; Merck KGaA); and rat anti-BrdU (1:500; cat no. B8434; Merck $\mathrm{KGaA})$. The fluorescent secondary antibodies and working concentrations were: Alexa Fluor-488 goat anti-mouse (1:500; cat no. A11001; Invitrogen; Thermo Fisher Scientific, Inc., Waltham, MA, USA) and cy3 goat anti-rat (1:500; cat no. 111-165-144; Jackson ImmunoResearch Laboratories, Inc., West Grove, PA, USA).

Western blotting. Rats were sacrificed 7 days post-WBI. Western blotting was conducted by deeply anesthetizing rats with an intraperitoneal injection of $1 \mathrm{ml} / 100 \mathrm{~g}(360 \mathrm{mg} / \mathrm{kg}$ body weight) chloral hydrate. Following the loss of the righting reflex, animals were stunned and the carotid artery and spinal cord was severed using sharp scissors. Brains were excised and transferred to ice-cold PBS, rinsed carefully, and dissected under a stereomicroscope under cold conditions with ice-cold PBS. The hippocampus of each hemisphere was separately analyzed, gently rinsed in ice-cold PBS and snap-frozen in liquid nitrogen. 
Western blotting was performed as previously described (7). The integrated densities of each band were quantified using Image J Software (version 2006.02.01; National Institutes of Health, Bethesda, MD, USA). Numerous exposures were obtained for each immunoblot to ensure that densitometry was performed on images captured within the linear exposure range. The primary antibodies and their dilutions were as follows: Mouse anti-DNMT 1 (1:1,000; cat no. ab13537; Abcam, Cambridge, UK); mouse anti-DNMT3A (1:1,000; cat no. ab13888; Abcam); mouse anti-DNMT3B (1:2,000; cat no. ab13604; Abcam) and anti- $\beta$-actin (1:10,000; cat no. A5316; Sigma-Aldrich; Merck KGaA). The secondary antibodies used were goat anti-mouse horseradish-peroxidase HRP $(1: 10,000$; cat no. 58307; Jackson ImmunoResearch Laboratories, Inc.).

Quantitative analysis. Stained tissue sections were evaluated by confocal microscopy, with split panel and z-axis analysis. Cell counts were evaluated in 5-10 tissue sections per rat using a multi-channel configuration with a x10 objective lens and $\mathrm{x} 10$ eyepiece. The cell counts were limited to regions in the granule cell layer and hilus. The volumes of the granule cell layer and hilus were used to normalize the numbers of $\mathrm{Brdu}^{+} / \mathrm{NeuN}^{+}$cells. For dendritic growth analysis, a series of images were acquired at $1-\mu \mathrm{m}$ intervals along the $\mathrm{z}$-section using a x10 lens and x10 eyepiece with a digital zoom of 2-3. Maximum intensity projections of the $\mathrm{z}$-series were applied to create merged images for the analysis.

Statistical analysis. All procedures were repeated 3 times. Data are presented as the mean \pm standard error of the mean. Statistical analyses were performed using one-way analysis of variance to compare the groups followed by a Kruskal-Wallis post hoc test. $\mathrm{P}<0.05$ was considered to indicate a statistically significant difference. All data were analyzed using SPSS 20.0 (IBM Corp., Armonk, NY, USA).

\section{Results}

WBI inhibits the proliferation of progenitor cells of hippocampus. Rats were sacrificed 7 days following WBI. Proliferating neurons were labeled with BrdU and NeuN $\left(\mathrm{BrdU}^{+} \mathrm{NeuN}^{+}\right)$; confocal microscopy was used to determine the number of $\mathrm{BrdU}^{+} \mathrm{NeuN}^{+}$cells. Irradiated rats exhibited a markedly lower number of new neurons compared with the control group. The number of $\mathrm{BrdU}^{+} \mathrm{NeuN}^{+}$cells was reduced by $71.8 \%(\mathrm{P}<0.01)$ in the radiation group compared with in the control group (Fig. 1).

WBI inhibits dendritic growth of novel neurons. The dendritic development of granule cells in the dentate gyrus is crucial for integrating into hippocampal circuits $(8,9)$. Thus, the length of dendrites was analyzed via immunofluorescence staining for DCX. The results of the present study indicated that, compared with that in the control rats, the total dendritic length of novel neurons was significantly less $(52.6 \%)$ in the irradiated group ( $\mathrm{P}<0.05$; Fig. 2).

DNMT1 and DNMT3A protein levels in the hippocampus following WBI. Western blotting revealed a 26.0 and $22.8 \%$ reduction in the protein levels of DNMT1 and DNMT3A, respectively, following irradiation $(\mathrm{P}<0.05$; Fig. 3). DNMT3B levels were unaltered following irradiation.

Zebularine decreases DNMT protein expression levels and inhibits precursor cell development. Zebularine is a DNA methylation inhibitor that increases the affinity of DNA-methyltransferase binding with DNA so that the former becomes inactive. To assess the effects of DNMTs on dendritic development, zebularine was utilized to determine whether the decrease in DNMT resulted in reduced cell proliferation. The results of the present study revealed that there were no variations between the zebularine-only and the radiation-only groups. Compared with that in the control group, radiation together with zebularine decreased DNMT1 (45.0\%) and DNMT3A (41.7\%) expression levels $(\mathrm{P}<0.05$; Fig. 3). Additionally, compared with that in the control group, radiation together with zebularine decreased the percentage of proliferative cells (90.3\%, $\mathrm{P}<0.05$; Fig. 1D and $\mathrm{E})$ and the total dendritic length (73.0\%, P<0.05; Fig. 2D and F).

Compared with the radiation-only group, the radiation and zebularine group exhibited lower DNMT1 protein levels (24.7\%) and DNMT3A (23.5\%). A more significant reduction in the percentage of proliferative cells (62.6\%; Fig. 1C and D) and total dendritic length (43.6\%; Fig. 2) was observed in the radiation + zebularine group than the radiation-only group $(\mathrm{P}<0.05)$.

\section{Discussion}

The present study investigated the effects of WBI on DNMT1, DNMT3A, and DNMT3B protein expression levels in the hippocampus and the function of these DNA methyltransferases in radiation-induced neurogenesis impairment. The findings of the present study revealed that radiation inhibited cellular proliferation, dendritic growth, and DNMT1 and DNMT3A protein expression levels in the hippocampus. Radiation and zebularine treatment exhibited a highly significant inhibitory effect compared with radiation treatment alone. These results indicated that DNMT1 and DNMT3A may be involved in the pathogenesis of WBI-induced neurogenesis. To the best of our knowledge, this is the first report of alterations in DNMT expression in the hippocampus of a rat model of radiation brain injury.

Cognitive impairment has been reported in patients irradiated for the treatment of head and neck cancer, as well as primary and secondary brain tumors (4). Irradiation of the temporal lobe, including the hippocampus, cannot usually be avoided during radiation therapy. Thus, patients are at an increased risk of hippocampal injury and impairment of complex cognitive processes, including spatial recognition (20) and declarative memory, which depend on the integrity of the hippocampus (21). The hippocampus has been recognized as a region associated with radiation-induced cognitive dysfunction $(5,20)$.

Gene expression studies have demonstrated that DNMTs in the central nervous system may be distinguished by their expression profiles. The presence of DNMT3B is only observed in neural progenitor tissue during early embryogenesis $(15,22)$. However, DNMT1 and DNMT3A expression levels are high within neurons during embryogenesis and 

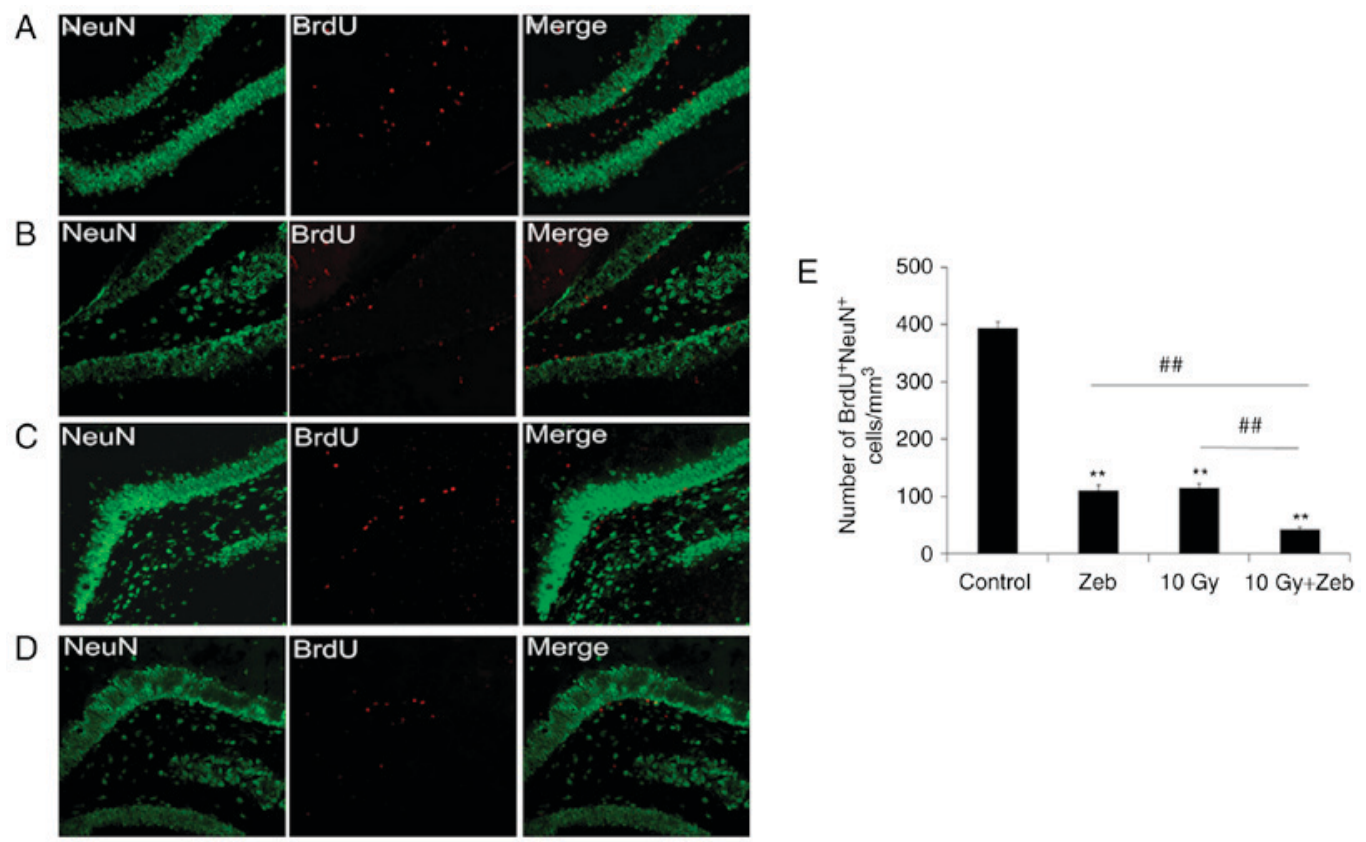

Figure 1. Proliferation analysis of neural precursor cells in the hippocampus. Confocal micrographs (magnification, $\mathrm{x} 40$ ) revealed novel neurons labeled for NeuN (green) and BrdU (red). (A) Control, (B) Zeb, (C) 10 Gy radiation, (D) 10 Gy radiation and Zeb and (E) quantification of the number of BrdU ${ }^{+} \mathrm{NeuN}^{+}$ cells in different groups. ${ }^{* *} \mathrm{P}<0.01$ vs. the control group. ${ }^{\# \#} \mathrm{P}<0.01$ vs. the $10 \mathrm{~Gy}+$ Zeb group. BrdU, bromo-deoxyuridine; control, no radiation exposure or Zeb injections; Zeb, zebularine; $10 \mathrm{~Gy}$, irradiated group; $10 \mathrm{~Gy}+\mathrm{Zeb}$, irradiated group treated with zebularine. NeuN, neuronal nuclear antigen.
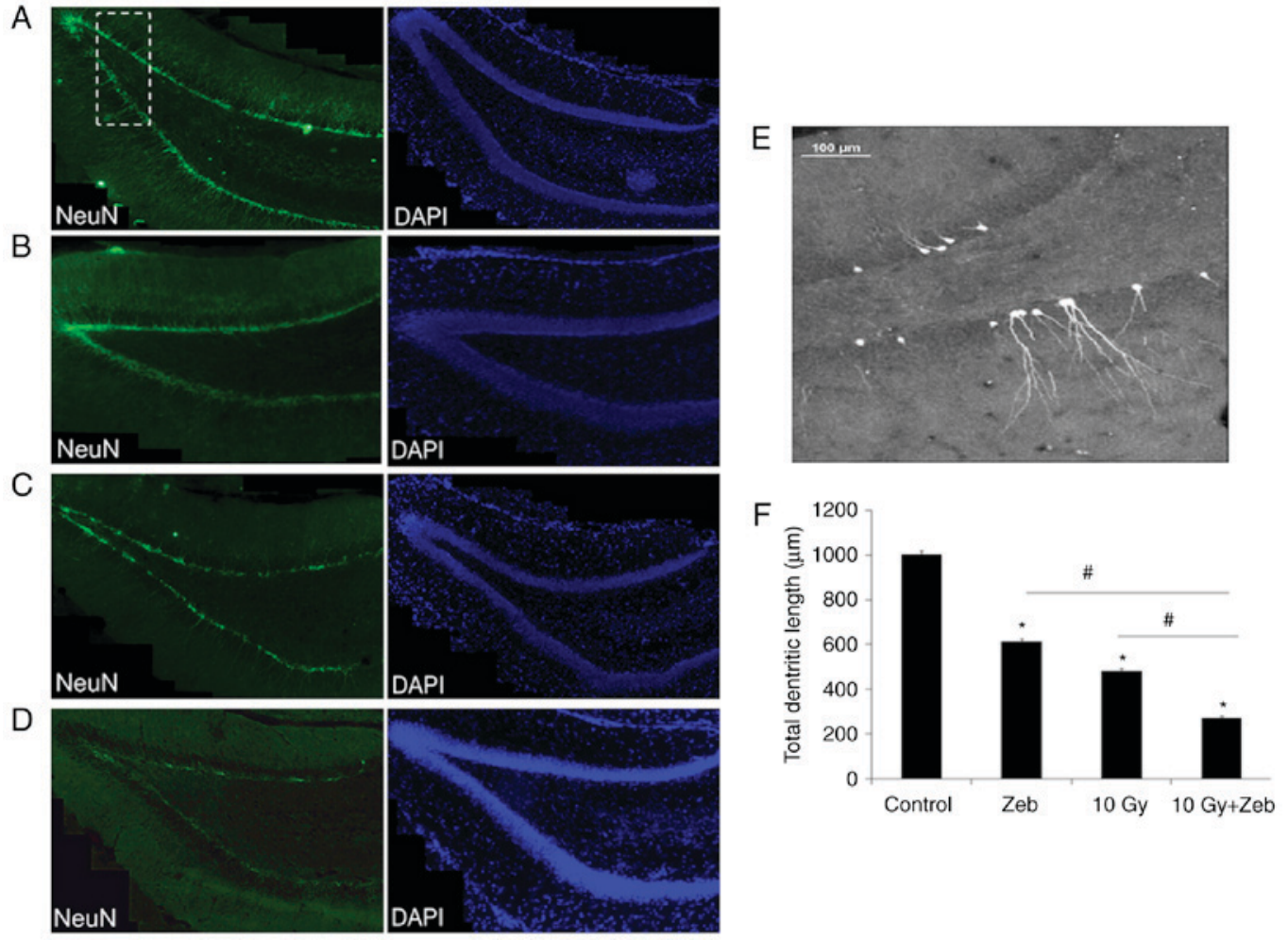

Figure 2. Dendrite length of newly formed neurons decreases following irradiation. DCX was used to label dendrites of novel neurons in the hippocampus (magnification, x20). Green, $\mathrm{DCX}^{+}$cells Blue, DAPI ${ }^{+}$cells. (A) Control, (B) Zeb, (C) 10 Gy radiation, (D) 10 Gy radiation and Zeb. (E) Magnified structure presented within the white dotted box in Fig. $2 \mathrm{~A}$. (F) Quantification. ${ }^{*} \mathrm{P}<0.05$ vs. the control group. ${ }^{~} \mathrm{P}<0.05$ vs. the 10 Gy + Zeb group. Control, no radiation exposure; DCX, doublecortin; Zeb, zebularine; $10 \mathrm{~Gy}$, irradiated group; $10 \mathrm{~Gy}+\mathrm{Zeb}$, irradiated group treated with zebularine.

throughout adulthood. This suggests that DNMTs and dynamic methylation states retain functional importance in the adult brain (22-24). Evidence revealed by Maddox et al (23) strongly indicates that DNMT activity regulates the consolidation and post-retrieval retention of alterations associated with conditioning in the tone-evoked neural activity of the lateral amygdala. In the developing mouse brain, deletion of DNMT1 in progenitor cells was associated 
A

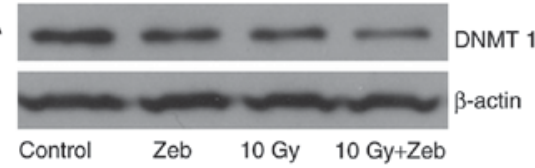

$\mathrm{B}$

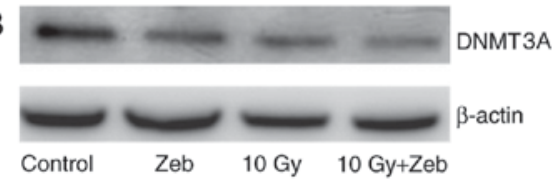

$\mathrm{C}$

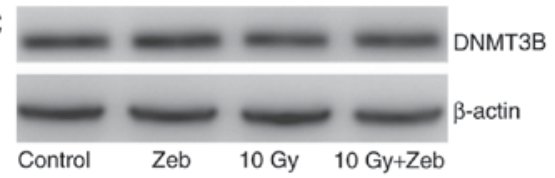

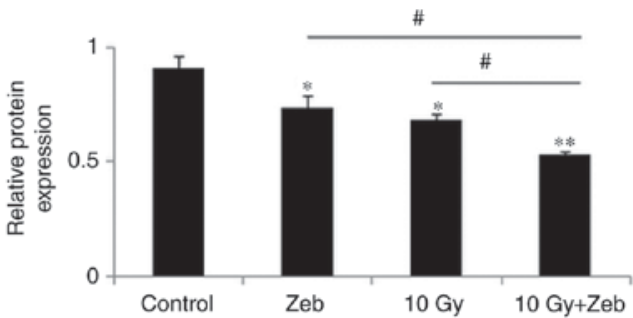
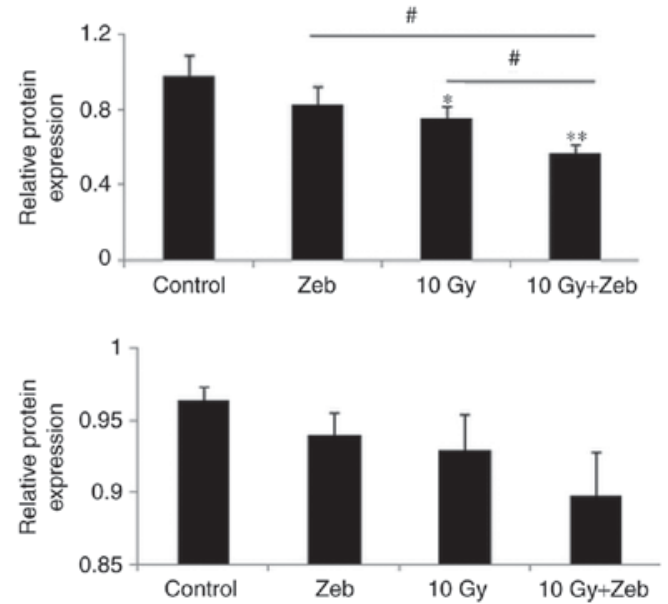

Figure 3. DNMT protein expression levels following irradiation. Western blot analysis demonstrated significantly lower protein expression levels of (A) DNMT1 and (B) DNMT3A following irradiation. Treatment with $10 \mathrm{~Gy}$ radiation and Zeb decreased DNMT1 and DNMT3A levels. Variation between the Zeb and 10 Gy groups were not observed. (C) No alterations were detected in the expression levels of DNMT3B. ${ }^{~} \mathrm{P}<0.05$ and ${ }^{* *} \mathrm{P}<0.01$ vs. the control group. ${ }^{*} \mathrm{P}<0.05$ vs. the $10 \mathrm{~Gy}+$ Zeb group. Control, no radiation exposure; DNMT, DNA methyltransferase; Zeb, zebularine; $10 \mathrm{~Gy}$, irradiated group; $10 \mathrm{~Gy}+$ Zeb, irradiated group treated with zebularine.

with the inhibition of neuronal maturation and survival (25). Singh and Thakur (24) demonstrated that age-associated memory decline was associated with lower levels of DNMT1 and higher levels of histone deacetylase 2. In the postnatal forebrain, DNMT3A is expressed in the subventricular zone and the hippocampal dentate gyrus $(26,27)$. Morris et al (26) demonstrated that DNMT3A-knockout mice exhibited synaptic alterations and learning deficits. These mutant mice lacking DNMT3A exhibited a loss of motor neurons in the hypoglossal nucleus and morphological defects in the neuromuscular junctions of the diaphragm. This indicated that DNMT3A could contribute to the survival of motor neurons and the maintenance of the neuromuscular endplate structure. Although the function of DNA demethylation in post-mitotic neurons remains uncertain, the possibility of an epigenetic mechanism that regulates cellular and behavioral activities requires further investigation.

Certain mental disorders in humans have been associated with DNA methylation (28). Immunodeficiency-centromere instability-facial anomalies syndrome is caused by a recessive mutation in the DNMT3B gene (28). Additionally, methyl $\mathrm{CpG}$ binding protein 2 gene mutations have been reported in Rett syndrome (29). The results of the present study demonstrated that DNMT1 and DNMT3A levels declined in the hippocampus following WBI, which may result in defective neurogenesis. The use of DNMT inhibitors also demonstrated that decreased expression of DNMTs inhibited the development of precursor cells. This finding is consistent with numerous studies $(17,18,26,30)$. Nelson et al (30) reported that DNMT inhibitors may cause deficits in excitatory synaptic transmission and reduce spontaneous network activity. Previously, the results of studies using DNMT inhibitors have indicated that DNA methylation may target specific genes involved in synaptic plasticity, as well as learning and memory $(17,23,31)$. Therefore, identifying the genes that are crucial to learning and memory that are also regulated by DNMT1 and DNMT3A may be beneficial to the understanding of human disorders.

All animals in the present study were male Sprague-Dawley rats; as it is commonly accepted that the emotions of female animals vary markedly during the different phases of the estrous cycle $(32,33)$. Furthermore, chromatin-modifying enzymes are regulated by estrogen (32). Tsai et al (33) demonstrated that epigenetic modifications, including DNA methylation and histone acetylation, varied between male and female mouse brains. Therefore, male rats were used throughout the present study to ensure consistency and provide data with high validity.

The present study demonstrated that WBI led to the impairment of neurogenesis and decreased the protein levels of DNMT1 and DNMT3A, and may be involved in the pathogenesis of WBI-induced cognitive deficits via the regulation of neuronal proliferation and dendritic growth.

\section{Acknowledgements}

The present study was supported by the National Natural Science Foundation of China (grant nos. 81402517 and 81372411), the Suzhou Science and Technology Project (grant no. SYS201651), the Suzhou Cancer Clinical Medical Center 
(grant no. Szzx201506), the Jiangsu Provincial Medical Youth Talent (grant no. QNRC2016234) and the Natural Science Foundation of Jiangsu Province (grant no. BK20171224).

\section{References}

1. Cairncross G, Wang M, Shaw E, Jenkins R, Brachman D Buckner J, Fink K, Souhami L, Laperriere N, Curran W and Mehta M: Phase III trial of chemoradiotherapy for anaplastic oligodendroglioma: Long-term results of RTOG 9402. J Clin Oncol 31: 337-343, 2013.

2. Xu T, Zhu G, He X, Ying H and Hu C: A phase III randomized study comparing neoadjuvant chemotherapy with concurrent chemotherapy combined with radiotherapy for locoregionally advanced nasopharyngeal carcinoma: Updated long-term survival outcomes. Oral Oncol 50: 71-76, 2014

3. Davis MA, Tyrrell J, Slotman GJ, Sudhindra R, Sachdeva K, Fanelle J, Smith G, Wurzer JV, Cassir J and Nazha NT; Southern New Jersey Head and Neck Cancer Treatment Network: Preoperative simultaneous fractionated cisplatin and radiation therapy in the treatment of advanced operable stage III and IV squamous cell carcinoma of the head and neck. Am J Surg 209: 575-579, 2015.

4. Douw L, Klein M, Fagel SS, van den Heuvel J, Taphoorn MJ, Aaronson NK, Postma TJ, Vandertop WP, Mooij JJ, Boerman RH, et al: Cognitive and radiological effects of radiotherapy in patients with low-grade glioma: Long-term follow-up. Lancet Neurol 8: 810-818, 2009.

5. Greene-Schloesser D, Moore E and Robbins ME: Molecular pathways: Radiation-induced cognitive impairment. Clin Cancer Res 19: 2294-2300, 2013.

6. Monje M and Dietrich J: Cognitive side effects of cancer therapy demonstrate a functional role for adult neurogenesis. Behav Brain Res 227: 376-379, 2012.

7. Ji S, Tian Y, Lu Y, Sun R, Ji J, Zhang L and Duan S: Irradiation-induced hippocampal neurogenesis impairment is associated with epigenetic regulation of bdnf gene transcription. Brain Res 1577: 77-88, 2014.

8. Bond AM, Ming GL and Song H: Adult mammalian neural stem cells and neurogenesis: Five decades later. Cell Stem Cell 17: 385-395, 2015

9. Park $\mathrm{H}$ and Poo MM: Neurotrophin regulation of neural circuit development and function. Nature Rev Neurosci 14: 7-23, 2013.

10. Cheng Z, Li YQ and Wong CS: Effects of aging on hippocampal neurogenesis after irradiation. Int J Radiat Oncol Biol Phys 94: 1181-1189, 2016.

11. Monje ML, Mizumatsu S, Fike JR and Palmer TD: Irradiation induces neural precursor-cell dysfunction. Nat Med 8: 955-962, 2002.

12. Marty VN, Vlkolinsky R, Minassian N, Cohen $\mathrm{T}$, Nelson GA and Spigelman I: Radiation-induced alterations in synaptic neurotransmission of dentate granule cells depend on the dose and species of charged particles. Radiat Res 182: 653-665, 2014

13. Fischer A, Sananbenesi F, Wang X, Dobbin M and Tsai LH: Recovery of learning and memory is associated with chromatin remodelling. Nature 447: 178-182, 2007.

14. Zovkic IB, Guzman-Karlsson MC and Sweatt JD: Epigenetic regulation of memory formation and maintenance. Learn Mem 20: 61-74, 2013.

15. Hon GC, Rajagopal N, Shen Y, McCleary DF, Yue F, Dang MD and Ren B: Epigenetic memory at embryonic enhancers identified in DNA methylation maps from adult mouse tissues. Nat Genet 45: 1198-1206, 2013

16. Miller CA, Gavin CF, White JA, Parrish RR, Honasoge A, Yancey CR, Rivera IM, Rubio MD, Rumbaugh $G$ and Sweatt JD: Cortical DNA methylation maintains remote memory. Nature Neurosci 13: 664-666, 2010.
17. Feng J, Zhou Y, Campbell SL, Le T, Li E, Sweatt JD, Silva AJ and Fan G: Dnmt1 and Dnmt3a maintain DNA methylation and regulate synaptic function in adult forebrain neurons. Nat Neurosci 13: 423-430, 2010.

18. LaPlant Q, Vialou V, Covington HE III, Dumitriu D, Feng J, Warren BL, Maze I, Dietz DM, Watts EL, Iñiguez SD, et al:Dnmt3a regulates emotional behavior and spine plasticity in the nucleus accumbens. Nat Neurosci 13: 1137-1143, 2010.

19. Tian Y, Shi Z, Yang S, Chen Y and Bao S: Changes in myelin basic protein and demyelination in the rat brain within 3 months of single 2-, 10-, or 30-Gy whole-brain radiation treatments. J Neurosurg 109: 881-888, 2008.

20. Pereira Dias G, Hollywood R, Bevilaqua MC, da Luz AC, Hindges R, Nardi AE and Thuret S: Consequences of cancer treatments on adult hippocampal neurogenesis: Implications for cognitive function and depressive symptoms. Neuro-oncol 16: 476-492, 2014.

21. Snyder JS, Soumier A, Brewer M, Pickel J and Cameron HA: Adult hippocampal neurogenesis buffers stress responses and depressive behaviour. Nature 476: 458-461, 2011.

22. Watanabe D, Uchiyama K and Hanaoka K: Transition of mouse de novo methyltransferases expression from Dnmt3b to Dnmt3a during neural progenitor cell development. Neurosci 142: 727-737, 2006.

23. Maddox SA, Watts CS and Schafe GE: DNA methyltransferase activity is required for memory-related neural plasticity in the lateral amygdala. Neurobiol Learn Mem 107: 93-100, 2014.

24. Singh P and Thakur MK: Reduced recognition memory is correlated with decrease in DNA methyltransferasel and increase in histone deacetylase 2 protein expression in old male mice. Biogerontology 15: 339-346, 2014.

25. Hutnick LK, Golshani P, Namihira M, Xue Z, Matynia A, Yang XW, Silva AJ, Schweizer FE and Fan G: DNA hypomethylation restricted to the murine forebrain induces cortical degeneration and impairs postnatal neuronal maturation. Hum Mol Genet 18: 2875-2888, 2009.

26. Morris MJ, Adachi M, Na ES and Monteggia LM: Selective role for DNMT3a in learning and memory. Neurobiol Learn Mem 115: 30-37, 2014

27. Simmons RK, Stringfellow SA, Glover ME, Wagle AA and Clinton SM: DNA methylation markers in the postnatal developing rat brain. Brain Res 1533: 26-36, 2013.

28. Hamidi T, Singh AK and Chen T: Genetic alterations of DNA methylation machinery in human diseases. Epigenomics 7: 247-265, 2015.

29. Lombardi LM, Baker SA and Zoghbi HY: MECP2 disorders: From the clinic to mice and back. J Clin Invest 125: 2914-2923, 2015.

30. Nelson ED, Kavalali ET and Monteggia LM: Activity-dependent suppression of miniature neurotransmission through the regulation of DNA methylation. J Neurosci 28: 395-406, 2008.

31. Wang H, Meyer K and Korz V: Stress induced hippocampal mineralocorticoid and estrogen receptor $\beta$ gene expression and long-term potentiation in male adult rats is sensitive to early-life stress experience. Psychoneuroendocrinology 38: 250-262, 2013.

32. Zhao Z, Fan L and Frick KM: Epigenetic alterations regulate estradiol-induced enhancement of memory consolidation. Proc Natl Acad Sci USA 107: 5605-5610, 2010.

33. Tsai HW, Grant PA and Rissman EF: Sex differences in histone modifications in the neonatal mouse brain. Epigenetics 4: 47-53, 2009.

This work is licensed under a Creative Commons Attribution-NonCommercial-NoDerivatives 4.0 International (CC BY-NC-ND 4.0) License. 